\title{
High Energy and Large Scale Structures as Gauge Invariant Spectra of Local Super-Symmetry
}

\author{
J. Towe \\ Department of Physics, Antelope Valley College, Lancaster, USA \\ Email: jtowe@avc.edu
}

How to cite this paper: Towe, J. (2018) High Energy and Large Scale Structures as Gauge Invariant Spectra of Local Super-Symmetry. Journal of Modern Physics, 9, 1144-1152.

https://doi.org/10.4236/jmp.2018.95069

Received: March 27, 2018

Accepted: April 27, 2018

Published: April 30, 2018

Copyright $\odot 2018$ by author and Scientific Research Publishing Inc. This work is licensed under the Creative Commons Attribution International License (CC BY 4.0).

http://creativecommons.org/licenses/by/4.0/ (c) (†) Open Access

\begin{abstract}
The least massive fermion generation is attributed to an analogue of Weyl curvature which occurs when a closed, spin- $2 \hbar$ string sweeps out a closed world tube: $W=\oint \frac{\mathrm{d} S}{\mathrm{~S}}$, where $S$ represents string length. A second order tube and consequent second order fermion mass are associated with a closed tube which circulates and itself sweeps out a closed tube: $W_{2}=2 ! \iint \mathrm{d} W \mathrm{~d} W$. Finally a $\mathrm{K}^{\text {th }}$ order tube and kth order fermion generation are associated with the general expression $W_{k}=k ! \iint \cdots \int \mathrm{d} W \mathrm{~d} W \cdots \mathrm{d} W$. By hypothesis six world tube orders establish an SU(3) symmetry and each closed tube interacts with a

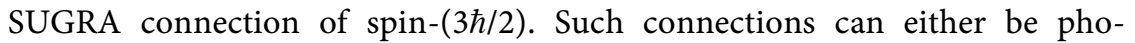
ton-fermion composites or composites that consist strictly of fermions. Interactions that involve no photons are, by hypothesis unobserved and are therefore associated with closed world tubes that are classified as dark mass-energy. It is demonstrated that interactions involving ordinary mass-energy are identities (e.g. interactions that are incapable of generating the proposed $\mathrm{SU}(3)$ symmetry). It is therefore concluded that dark mass-energy is a necessary condition for the SU(3) symmetry that by hypothesis characterizes the proposed model. Since $95 \%$ of the mass-energy in the universe is regarded as dark, the total mass-energy that constitutes the proposed SU(3) symmetry can be calculated as $\sum_{k=1}^{6}[0.95]\left[Q_{L} \psi_{L} \bar{l}_{R}\right]_{k}$, where $Q_{L}$ is a left-handed quark, where $\psi_{L}$ is a left-handed spin $2 \hbar$ particle and where $\bar{l}_{R}$ is a right-handed anti-lepton. Thus the mass-energy that is associated with the wave $\Phi=\exp \sum_{k=1}^{6}(0.95)\left[Q_{L} \psi_{L} \bar{l}_{R}\right]_{k}$ is about $10^{67} \mathrm{GeV} / \mathrm{c}^{2}$ (the approximate
\end{abstract}


mass of a typical galaxy). This wave is regarded by hypothesis as a single galactic unit and as the ground state of a large-scale quantization; i.e. as the ground state of a series of abstract waves which mimic de Broglie waves in the sense that the ground state is a wave of one anti-node which oscillates about a wave length that parallels a geodesic on a smallest abstract spherical shell. The first excited state is a wave of two anti-nodes that oscillate about a wave length that parallels a geodesic on the second smallest spherical shell etc. The proposed quantization is calibrated by a boundary condition which is established by observation and which separates galaxies by distances that are about ten times the diameter of a typical galaxy; separates clusters by distances that are about ten times the diameter of a typical cluster etc. Thus the wave $\Phi$ is, by hypothesis, the first in a series of gauge transformations $2^{n} \exp \{2 \pi(i) n \beta\}^{n} \cong 2^{n}\left\{\exp \frac{1}{0.434 \cdots}\right\}^{n}$ that models the galactic $=2^{n} 10^{n}=(20)^{n}: n=1,2, \cdots ; \beta=1 / 2 \pi(i)[0.434 \cdots]$

hierarchy. By hypothesis the above gauge transformations form an irreducible representation of the $\mathrm{SU}(3)$ symmetric hierarchy of fermionic generations that is introduced above, providing that tensor products are replaced by the six increments which correspond to the proposed gauge transformations. By hypothesis the local cluster (the state corresponding to $n=2$ ) results from the dispersion of those galaxies that originally form the 2 -spherical area by which the local cluster is bounded. This number of galaxies is said to be at least 54 . Thus the radius of the concentric sphere which forms the original 2-dimensional boundary of the local cluster is approximately $R=[54 \text { galaxies } / 4 \pi]^{1 / 2}=2.07$ mean radial units. Accordingly the area of the concentric sphere that corresponds to $n=6$ is approximately $A=4 \pi\left\{2.07 \times\left(20^{4}\right)\right\}^{2}=1.37 \times 10^{12}$ galactic units; or if one predicts 79 galaxies for the local cluster, then the proposed model indicates a universe of approximately $2 \times\left(10^{12}\right)$ galaxies, as established by the C. Conselice team at the University of Nottingham and by NASA.

\section{Keywords}

Closed World Tubes, Fermion Generations, Gauge Transformations, Galactic Hierarchy

\section{Introduction}

To discuss super-gravitational interactions it is necessary to review Osp(1/4)-pure super-gravity on $\mathrm{M}_{4}$. The Lagrangian density

$$
\mathcal{L}=\sqrt{-g} R+e \bar{\psi}_{\mu} \gamma_{v} \gamma^{5} \nabla_{\sigma} \psi_{\rho} \varepsilon^{\mu v \rho \sigma}
$$

is regarded as dual to the string background $\mathrm{AdS}_{7} \mathrm{XS}^{4}$ [1] [2]. The Lagrangian density (1) is based upon the super-Poincare algebra

$$
\left[M_{A}, M_{B}\right\}=f^{C}{ }_{A B} M_{C},
$$


where $M_{A}=\left(P_{a},-i M_{a b}, Q_{\alpha}\right)$. The $P_{a}$ represent the translation group, the $-i M_{a b}$ constitute the adjoint representation of the Lorentz group and the $Q_{\alpha}$ are components of the SUSY generator. The $\omega_{\mu}^{A}$ describe all connection fields

$$
\omega_{\mu}{ }^{A}=\left(e_{\mu}{ }^{a}, \omega_{\mu}{ }^{a b}, \bar{\xi}_{\mu}{ }^{\alpha}\right)
$$

and transform under $\mathrm{Osp}(1 / 4)$ as

$$
\delta \omega_{\mu}{ }^{A}=f^{A}{ }_{B C} \varepsilon^{B} \omega_{\mu}{ }^{C}
$$

The covariant derivative is

$$
\nabla_{\mu}=\partial_{\mu}+M_{A} \omega^{A}{ }_{\mu}=\partial_{\mu}+i \omega^{a b}{ }_{\mu} M_{a b}+\bar{\xi}_{\mu}{ }^{\alpha} Q_{\alpha}
$$

and the curvature tensor is derived from $\left[\nabla_{\mu}, \nabla_{v}\right]=R_{\mu \nu}^{A} M_{A}$, where

$$
R_{\mu \nu}{ }^{A}=\partial_{\nu} \omega^{A}-\partial_{\mu} \omega^{A}+f^{A}{ }_{B C} \omega_{\mu}{ }^{B} \omega_{v}{ }^{C}
$$

[3].

\section{Fermion Mass as Curvature}

Paralleling Wheeler's concept which attributes mass to space-time curvature [4], fermion generations are modeled as analogues of Weyl curvature states

$$
W=\oint \frac{\mathrm{d} S}{S}
$$

[5], which occur when closed, spin-2 strings sweep out closed world tubes. Based upon a hypothesis introduced by $F$. London [6], a curvature state (7) will be regarded as admissible if and only if gauge is preserved in the sense that

$$
\exp \oint \frac{\mathrm{d} S}{S}=\exp \{2 \pi(i) n\}: n=1,2, \cdots
$$

[6].

The proposed model which associates W-curvature with $W^{k}=k ! \int . . \int d W . . d W$ is calibrated by the association of $\mathrm{W}$-curvature $W^{6}$ with the mass of the heavy fermion generation. Specifically, calibration of the proposed model associates the curvature state $W^{\sigma}$ with the mass of the composite $\left(t_{L} \psi_{L} v^{\tau^{+}}\right)$where $t_{L}$ represents a left-handed top quark, where $\psi_{L}$ represents a left-handed spin- $2 \hbar$ field and where $v^{\tau^{+}}{ }_{R}$ represents an RH anti-tauon's neutrino:

$$
\operatorname{mass}\left(W^{6}\right)=6 !(0.25) \mathrm{GeV} / \mathrm{c}^{2}=173 \mathrm{GeV} / \mathrm{c}^{2}
$$

which is the generational partner of the top quark. It should be noted that the mass of the tauon's neutrino is negligible compared with that of the top quark, so that the mass (9) is essentially that of the top quark. In this context the masses of the other fermionic generations are identified as the masses of composites

$$
\begin{aligned}
& \operatorname{mass}\left(W^{5}\right)=5 !(0.25) \mathrm{GeV} / \mathrm{c}^{2}=29 \mathrm{GeV} / \mathrm{c}^{2} \\
& \operatorname{mass}\left(W^{4}\right)=4 !(0.25) \mathrm{GeV} / \mathrm{c}^{2}=6 \mathrm{GeV} / \mathrm{c}^{2} \\
& \operatorname{mass}\left(W^{3}\right)=3 !(0.25) \mathrm{GeV} / \mathrm{c}^{2}=1.5 \mathrm{GeV} / \mathrm{c}^{2}
\end{aligned}
$$




$$
\operatorname{mass}\left(W^{2}\right)=2 !(0.25) \mathrm{GeV} / \mathrm{c}^{2}=0.5 \mathrm{GeV} / \mathrm{c}^{2}
$$

and

$$
\operatorname{mass}(W)=(0.25) \mathrm{GeV} / \mathrm{c}^{2}=0.25 \mathrm{GeV} / \mathrm{c}^{2}
$$

The theoretically established masses other than (10) are approximately equal to observationally established values [7] [8]. Expression (10) predicts a new quark of mass $29 \mathrm{GeV} / \mathrm{c}^{2}$ and of $I_{3}=-1 / 2$, which is interpreted as the generational partner of the muon.

In cases other than (11), lepton masses are relatively negligible. Thus the listed masses closely approximate the masses of the associated quarks. Expression (11) is associated with the sum of the mass of the bottom quark approximately 4.3 $\mathrm{GeV} / \mathrm{c}^{2}$ and that of the tauon, approximately $1.7 \mathrm{GeV} / \mathrm{c}^{2}$. Expression (12) is associated with the mass of the charmed quark; expression (13) essentially represents the mass of the strange quark and (14) essentially represents the mass of the average quark. An average quark is characterized by the quantum numbers that result when the masses, the electrical charges, the $I_{3}$ numbers etc. of the up and down quarks are averaged. The average quark is complemented by an average lepton which is characterized by the quantum numbers that result when the electrical charges, the $I_{3}$ numbers etc. of the electron and those of its neutrino are averaged.

The spin- $2 \hbar$ composites (9) through (14) constitute an SU(3) symmetry which is illustrated by Figure 1 .

The vertex labeled " $173 \mathrm{GeV} / \mathrm{c}^{2}$ " represents the mass of the most massive fermion generation, which is described in the proposed model by the notation $\left(t_{L} \psi_{L} v_{R}^{\tau^{+}}\right)$, where $t_{L}$ represents a left-handed top quark, where $\psi_{L}$ represents a left-handed spin- $2 \hbar$ particle and where $v_{R}{ }^{{ }^{+}}$represents a right-handed anti-tauon's neutrino which is regarded, by hypothesis as the generational partner of the top quark. The vertex labeled " $29 \mathrm{GeV} / \mathrm{c}^{2}$ " represents the mass of the second most massive fermion generation, which is described in the proposed model by the notation $\left(7_{L} \psi_{L} \mu_{R}^{+}\right)$, where $7_{L}$ represents a left-handed quark that is predicted by the proposed model, where $\psi_{L}$ represents a spin- $2 \hbar$ particle and where $\mu_{R}^{+}$represents a right-handed anti-muon, which is regarded by hypothesis as the generational partner of the $7_{R}$ quark. The vertex labeled " $6.0 \mathrm{GeV} / \mathrm{c}^{2}$ " represents the mass of the third most massive fermion generation, which is described in the proposed model by the notation $\left(b_{L} \psi_{L} \tau_{R}^{+}\right)$, where $b_{L}$ represents a left-handed bottom quark, where $\psi_{L}$ represents a left-handed spin- $2 \hbar$ particle, and where $\tau_{R}{ }^{+}$represents a right-handed anti-tauon, which is regarded, by hypothesis as the generational partner of the $b_{L}$ quark. The vertex labeled " $1.5 \mathrm{GeV} / \mathrm{c}^{2 \text { " }}$ represents the mass of the fourth most massive fermion generation which is described in the proposed model by the notation $c_{L} \psi_{L} v_{R}^{\mu^{+}}$, where $c_{L}$ represents a left-handed charmed quark, where $\psi_{L}$ represents a left-handed spin-2 $\hbar$ particle and where $v_{R}^{\mu^{+}}$ represents the right-handed anti-muon's neutrino. The vertex labeled $0.5 \mathrm{GeV} / \mathrm{c}^{2}$ 
represents the mass of the fifth most massive fermion generation which is described in the proposed model by the notation $\left(s_{R} \psi_{L} e_{L}^{+}\right)$, where $s_{R}$ represents the right-handed strange quark, where $\psi_{L}$ is a spin- $2 \hbar$ particle and where $e_{L}^{+}$ represents a left-handed anti-electron. The vertex labeled $0.25 \mathrm{GeV} / \mathrm{c}^{2}$ represents the mass of the least massive fermion generation, which is described in the proposed model by the notation $\left(A_{L} \psi_{L} \bar{a}_{R}\right) . A_{L}$ represents a left-handed average quark $\psi_{L}$, represents a left-handed particle of spin- $2 \hbar$ and $\bar{a}_{R}$ represents a right-handed average electron.

In the proposed model, the above-described generations are regarded as

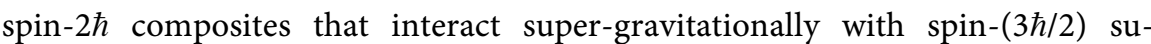
per-gravitational connections which are described in Section 1.

By hypothesis there are two classes of super-gravitational interactions between the proposed, spin- $2 \hbar$ world tubes and the SUGRA connections which are discussed in Section 1. In one class the connections are composed exclusively of fermions.

$$
t_{L} \psi_{L} v_{R}^{\tau^{+}}+\bar{t}_{R} \bar{c}_{R} v_{R}^{\tau^{+}} \rightarrow \bar{C}_{R} \psi_{L} v_{L}^{\mu^{-}}+v_{R}^{\tau^{+}} v_{R}^{\tau^{+}} v_{R}^{\mu^{+}}
$$

and

$$
v_{R}^{\tau^{+}} v_{R}^{\tau^{+}} v_{R}^{\mu^{+}}+\bar{C}_{R} \psi_{L} v_{L}^{\mu^{-}} \rightarrow t_{L} \psi_{L} v_{R}^{\tau^{+}}+\bar{t}_{R} \bar{C}_{R} v_{R}^{\tau^{+}} .
$$

In the other class each connection is a composite of a fermion and a photon:

$$
\gamma_{R} \bar{t}_{R}+c_{L} \psi_{L} v_{R}{ }^{\mu^{+}} \rightarrow c_{L} \psi_{L} v_{R}{ }^{\mu^{+}}+\gamma_{R} \bar{t}_{R}
$$

and

$$
\gamma_{R} \bar{t}_{R}+c_{L} \psi_{L} \nu_{R}^{\mu^{+}} \rightarrow c_{L} \psi_{L} \nu_{R}^{\mu^{+}}+\gamma_{R} \bar{t}_{R} .
$$

Because the former class of interactions (that described by the Equations (15)) does not involve absorption or radiation of photons, the spin- $2 \hbar$ composites with which spin- $(3 \hbar / 2)$ connections interact are not visible. These spin- $2 \hbar$ composites are therefore classified as dark mass-energy. Upon inspection it becomes evident that the interactions represented by the equations (16) cannot generate the proposed, $\mathrm{SU}(3)$ symmetry. In the proposed model then, the dark mass-energy which is involved in SUGRA interactions such a (15) is established as a necessary condition for the SU(3) symmetry of fermion generations which is depicted by Figure 1 .

\section{Quantization of the Large Scale}

According to observation $95 \%$ of observed mass-energy is dark. Consequently a "dark metric"

$$
\sum_{k=1}^{6}\left[(0.95) Q_{L} \psi_{L} \bar{l}_{R}\right]_{k}
$$

is applied to the $\mathrm{SU}(3)$ symmetric hierarchy of fermionic generations that are involved in the interactions (15). Thus the wave equation

$$
\exp \sum_{A=1}^{6}(0.95 W)^{A}
$$




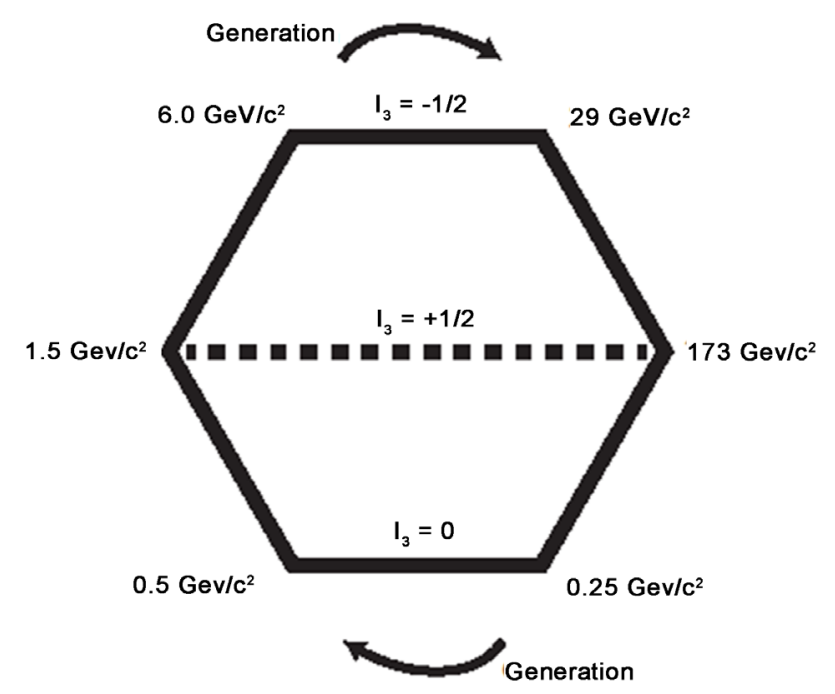

Figure 1. The SU(3) symmetry which is constituted by the world tubes (9) through (14).

is associated with mass-energy

$$
\begin{aligned}
& \exp \left\{(0.95)^{6} W^{6}+(0.95)^{5} W^{5}+(0.95)^{4} W^{4}\right. \\
& \left.+(0.95)^{3} W^{3}+(0.95)^{2} W^{2}+(0.95) W\right\}
\end{aligned}
$$

or

$$
\begin{aligned}
& \exp \{(0.73)(173)+(0.77)(29)+(0.81)(6.0)+(0.86)(1.5) \\
& +(0.90)(0.5)+(0.95)(0.25)\}
\end{aligned}
$$

or

$$
\exp \{127+22+4.9+1.3+0.45+0.24\} \cong 10^{67} \mathrm{GeV} / \mathrm{c}^{2}
$$

which is approximately the mass of a typical galaxy. This mass is regarded by hypothesis as one galactic unit and as the ground state of a large scale quantization.

By hypothesis the wave (21) is the ground state of an abstract series of waves which mimic de Broglie waves in the sense that the ground state is a wave of one anti-node which oscillates about a wave length which parallels a geodesic on the smallest in a set of abstract, concentric, spherical shells. The first excited state is a wave of two anti-nodes that oscillates about a segment that parallels a geodesic on the second smallest in the set of abstract, concentric, spherical shells etc.

This model is motivated by the hypothesis that each gauge transformation 22) on the wave (18) produces an inflation event resulting in a space-like, 3-volume that is bounded by a 2 -spherical area which is populated by mass-energy. (This concept should be compared with the observations of Huchra and Geller at the Harvard-Smithsonian Center for Astrophysics [9].) This 2-distribution of mass-energy is gradually modified as mass-energy disperses toward a condition of 3-dimensional homogeneity and isotropy. By hypothesis this process is repeated by a second gauge transformation and consequent inflation event etc.

The proposed quantization is calibrated by a boundary condition (established 
by observation) which separates galaxies by distances that are about ten times the diameter of a typical galaxy; separates clusters by distances that are about ten times the diameter of a typical cluster etc. In this context the gauge transformations which by hypothesis occur to the wave (18) are as follows:

$$
\begin{aligned}
& 2^{n} \exp \{2 \pi(i) n \beta\}^{n} \cong 2^{n}\left[\exp \frac{1}{0.434 \cdots}\right]^{n} \\
& \cong 2^{n} 10^{n}=(20)^{n}: n=1,2, \cdots ; \beta=1 / 2 \pi(i)[0.434 \cdots]
\end{aligned}
$$

This is an abstract model in which ever larger concentric shells represent ever larger quantized levels of the galactic hierarchy. As stated above, the calibrating number that is associated with the area of the $n=1$ state of (22) is a single galaxy. The area which forms the boundary of the $n=2$ state is regarded as the same as the number of galaxies that populates the $n=2$ state after the 2-dimensional boundary has disbursed into the 3-dimensional configuration that is known as the local cluster. This number of Galaxies is said to be at least 54 .

The question that now arises is what is the largest 2-spherical area that results from the gauge transformations (22)? The answer is provided by an algebraic hypothesis: The gauge transformations (22) are cumulatively regarded as forming an irreducible representation of the $S U(3)$ symmetric hierarchy of fermionic generations (Figure 1), where tensor products are replaced by the six increments

$$
R_{1}\{(2)(10)\}^{n}: n=1,2, \cdots, 6
$$

where $R_{1}$ is the radius of the ground state $n=1$. In this context the largest area to be obtained from a gauge transformation (22) is produced from four gauge transformations that are applied to the local cluster (the $n=2$ state). According to observation the number of galaxies in the local cluster, which is interpreted as the area of a concentric sphere $(n=2)$ is "more than 54". This number can be implemented to calculate the radius of the local cluster:

$$
R=[54 \text { galaxies } / 4 \pi]^{1 / 2}=2.07 \text { mean radial units. }
$$

According to the boundary condition upon which Equation (22) is founded, the scale factor $(20)^{\wedge} 4$ transforms an observer of the local cluster (the state $n=2$ ) into an "observer" of the universe that results from the sixth gauge transformation (the fourth gauge transformation subsequent to the establishment of the local cluster). Thus the radius of the local cluster is transformed into 331,200 mean radial units. Consequently Equation (23) can again be implemented to establish the number of galaxies in the universe; i.e. the number that results from the sixth gauge transformation: $A=4 \pi\left[(2.07)(20)^{4}\right]^{2}$ or

$$
A=4 \pi R^{2}=1.37 \times 10^{12} \text { galaxies } .
$$

According to NASA and the A. Conselisce team however, there are at least two trillion galaxies in the universe [10]. According to the structure indicated by the proposed model this value indicates that 79 galaxies populate the local cluster. To confirm that this number corresponds, in the proposed model to the two 
trillion galaxies that are indicated by current research, let us consider the expression $R=[79 \text { galaxies } / 4 \pi]^{1 / 2} \cong 2.51$ mean radial units. In this context

$$
R(20)^{4}=(2.51)(20)^{4} \cong 401600 \text { mean radial units. }
$$

Thus

$$
A=4 \pi r^{2}=4 \pi(401600)^{2} \text { galaxies } \cong 2.03 \times 10^{12} \text { galaxies } .
$$

\section{Summary}

The least massive fermion generation was attributed to an analogue of Weyl curvature which occurs when a closed, spin-2 string sweeps out a closed world tube: $W=\oint \frac{\mathrm{d} S}{\mathrm{~S}}$. A second order tube and consequent second order fermion mass were associated with a closed tube which circulates and itself sweeps out a closed tube: $W_{2}=2 ! \iint \mathrm{d} W \mathrm{~d} W$. Finally a $\mathrm{K}^{\text {th }}$ order tube and kth order fermion generation were associated with a configuration $W_{k}=k ! \iint \cdots \int \mathrm{d} W \mathrm{~d} W \cdots \mathrm{d} W$. By hypothesis six world tube orders established an $\mathrm{SU}(3)$ symmetry and each closed tube interacted with SUGRA connections of spin-(3ћ/2). Such connections can either be photon-fermion composites or composites that consist strictly of fermions. Interactions that involve no photons are, by hypothesis unobserved and are therefore associated with closed world tubes that are classified as dark mass-energy. It was demonstrated that interactions involving ordinary mass-energy are identities (e.g. interactions that are incapable of generating the proposed $\mathrm{SU}(3)$ symmetry). Based upon this observation it is concluded that dark mass-energy is a necessary condition for the SU(3) symmetry that by hypothesis characterizes the proposed model. Since $95 \%$ of the mass-energy in the universe is regarded as dark, the total mass-energy that constitutes the proposed $\mathrm{SU}(3)$ symmetry was calculated as $\sum_{k=1}^{6}[0.95]\left[Q_{L} \psi_{L} \bar{l}_{R}\right]_{k}$. Thus the mass-energy that is associated with the wave $\Phi=\exp \sum_{k=1}^{6}(0.95)\left[Q_{L} \psi_{L} \bar{l}_{R}\right]_{k}$ is about $10^{67}$ $\mathrm{GeV} / \mathrm{c}^{2}$ (the approximate mass of a typical galaxy). This wave was therefore regarded by hypothesis as a single galactic unit and as the ground state of a large-scale quantization; i.e. as the ground state of a series of abstract waves which mimic de Broglie waves in the sense that the ground state is a wave of one anti-node which oscillates about a wave length that parallels a geodesic on the smallest abstract spherical shell. The first excited state is a wave of two anti-nodes that oscillate about a wave length that parallels a geodesic on the second smallest spherical shell etc. The proposed quantization is calibrated by a boundary condition which is established by observation and which separates galaxies by distances that are about ten times the diameter of a typical galaxy; separates clusters by distances that are about ten times the diameter of a typical cluster etc. Thus the wave $\Phi$ is the first in a series of gauge transformations

$$
\begin{aligned}
& 2^{n} \exp \{2 \pi(i) n \beta\}^{n} \cong 2^{n}\left\{\exp \frac{1}{0.434 \cdots}\right\}^{n} \\
& =2^{n} 10^{n}=(20)^{n}: n=1,2, \cdots ; \beta=1 / 2 \pi(i)[0.434 \cdots]
\end{aligned}
$$


that models the galactic hierarchy. Proposed gauge transformations $\left(^{*}\right)$ form an irreducible representation of the SU(3) symmetric hierarchy of fermionic generations that was initially introduced, providing that six tensor products are replaced by six increments $R_{1}\{(2)(10)\}^{n}: n=1,2, \cdots, 6$, which correspond to the gauge transformations $\left(^{*}\right)$ and that $R_{1}$ is the radius of the ground state $n=1$. The local cluster (the state corresponding to $n=2$ ) results by hypothesis from the dispersion of those galaxies that originally form the area of this second smallest concentric sphere. This number of galaxies is said to be at least 54 . Thus the radius of the concentric sphere which corresponds to $n=2$ is approximately $R=[54 \text { galaxies } / 4 \pi]^{1 / 2}=2.07$ mean radial units. Accordingly the area of the concentric sphere that corresponds to $n=6$ is approximately $A=4 \pi\left\{2.07 \times\left(20^{4}\right)\right\}^{2}=1.37 \times 10^{12}$ galactic units; or if one predicts 79 galaxies for the local cluster, then the proposed model indicates approximately $2 \times\left(10^{12}\right)$ galaxies in the universe, as established by the C. Conselice team at the University of Nottingham and by NASA.

\section{Acknowledgements}

Figure 1 was designed by R. James Towe.

\section{References}

[1] D'Hoker, E. and Freedman, D. (2002) Super-Symmetric Gauge Theories and the AdS/CFT Correspondance. arXiv.hep-th/0201253

[2] Aharony, O., Gubser, S., Maldacina, J., Ooguri, H. and Oz, Y. (2000) Physics Reports, 323, 183-386. https://doi.org/10.1016/S0370-1573(99)00083-6

[3] Kaku, M. (1993) Quantum Field Theory. Oxford University Press, New York, 693.

[4] Wheeler, J.A. (1962) Curved Empty Space as the Building Material of the Physical World. In: Nagel, E., Suppes, P. and Tarski, A., Eds., Logic, Methodology and the Philosophy of Science, Stanford University Press, Stanford.

[5] Weyl, H. (1922) Space, Time and Matter. Metheun and Company Ltd., London, Ch. 4, Section 35 .

[6] London, F. (1927) Zeitschrift fur Physik, 42, 375-389. https://doi.org/10.1007/BF01397316

[7] SLAC Virtual Visitor Center (2009) SLAC National Accelerator Laboratory. Menlo Park.

[8] Bock, R. and Vaselesca, A. (1999) Particle Detector BriefBook. Springer, Berlin.

[9] Huchra, J.P. and Geller, M. (1987) Semi-Annual Report. Harvard-Smithsonian Observatory, 1 July-31 December.

[10] Conselisce, C., Wilkenson, A., Duncan, K. and Mortlock, A. (2016) The Astrophysical Journal, 830, Article ID: 83. https://doi.org/10.3847/0004-637X/830/2/83 